\title{
An Effective Respiration Signal Processing System Based on improved EEMD Method and PPG
}

\author{
WangTao ${ }^{\mathrm{a}}, \mathrm{Zhu} \mathrm{Xi}^{\prime} \mathrm{An}^{\mathrm{b}}$ and Liu JiZe ${ }^{\mathrm{c}}$ \\ ${ }^{1,2}$ School of Information and Communication Engineering, Beijing Information Science and \\ Technology University, Beijing 100101, China; \\ awangtaobistu@foxmail.com, bzhuxian1962@163.com, c448091315@qq.com
}

\section{Keywords:Improved EEMD, PPG,DSP}

\begin{abstract}
Aneffective signal processingsystem based on improved Ensemble Empirical Mode Decomposition(EEMD) method is proposed for the analysis of extracting respiration from Photoplethysmography(PPG).The proposed improved EEMD method adaptively adds the noise intensity by calculating the average sub-peak amplitude of PPG.Moreover,an attempt ismade to increase the efficiency of the computational process by reducing the number of EMD cycles using the $S$ number convergence criterion based on characteristics of PPG.The proposed algorithm is examined by the on-board DSP and derives better results.It is helpful for non-stationary biomedicalsignal processing.
\end{abstract}

\section{Introduction}

Respiration is one of the important vital signs of human body as well as a primary and continuous detectingparameter of the critically ill patients.Photoplethysmography (PPG) has been widely used for respiration extraction due to its advantages, such as less expensive, non-invasive, long time monitoring, and easy operation.It has been intensivelyinvestigated by researchers in this respect.

Discrete Wavelet Transform(DWT) is proposed to extract the respiration depends on the selection of the wavelet basis, the number of layers, and the threshold[1].Wave coefficient between reconstructed respiration and original respiration based on Empirical Mode Decomposition(EMD)is around 0.4.The EMD method providesanadaptive basis for the change of the signal to improve the deficiency of wavelet analysis,which decomposes signals into finite sets of Intrinsic Mode Functions (IMF).The IMFs cause a serious deviation in the frequency domain distribution of the signal and confusion about the physical meaning because of mode mixing problem[2].The Ensemble Empirical Mode Decomposition is proposed to effectively overcome the problem of EMD,but the intensity of the noise intensity that seriously affect the extraction effect and program running time is not given according to the feature of PPG signal[3].

In this paper, we propose animproved EEMD method whichadaptively determines the noise intensity according to the average sub-peak amplitude of the PPG signalwhilethe minimumcycles of extracting extreme value is also ascertained.The purposeof thestudy is to provide an adaptive additive noise of EEMD method and to examine the possibilities of hardwareimplementation forextracting respiratory from the PPG signal.

\section{A BRIEF REVIEW OF THE EEMD METHOD}

EEMD is a kind of adaptive signal analysis method based on local extreme value of signal,it decomposes a signal S(t) into a series of IMFs, which overcome the mixed problem of EMD mode.

Fig. 1 shows the data flow of the EEMD method. A step by step description is summarized briefly as follows:

(1)Add white noise with0.2 times the intensity of the signal standard deviation.

(2) Calculate all the local maximum and local minimum ofS(t) and generate upper and lower envelopesby cubic spline fitting.

(3) Calculate the mean envelope,m(t), by averaging the upperand lower envelopes. 
(4) Calculateh $_{1}(\mathrm{t})=\mathrm{S}(\mathrm{t})-\mathrm{m}(\mathrm{t})$.

(5) If a convergence criterion is satisfied, then IMF is derived;Otherwise,record IMF and go to (1).

(6) Acquire the residue and checkif it is a monotonic functionor a constant.

(7) Until reachingthe number of noise trials,average allthe corresponding IMFs.

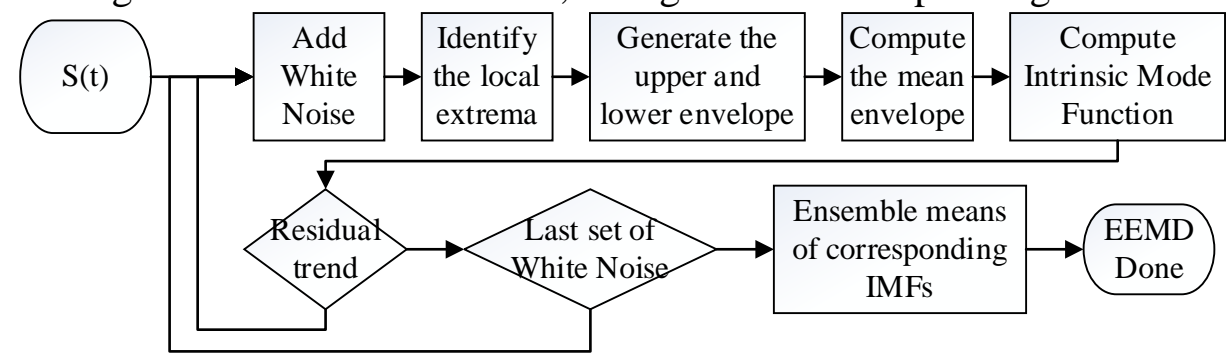

Fig.1 Data flow of EEMD method

Forthe standard EMD process, the aforementioned convergence criterion in step (5) reads:the residuals are monotonic functions.In this paper, we adopt the $S$ number criterion instead. For it is the simplest,hence, themost suitable one for hardwareimplementation.However, the best numberof $S$ for different signal is not available[4].To test the effect ofthe choice of Son the reconstructed respirationwave,we plot the correlation coefficients of the respiration and IMF7, for different values of S.IMF7 is chosen because it relates the most to the respiration.

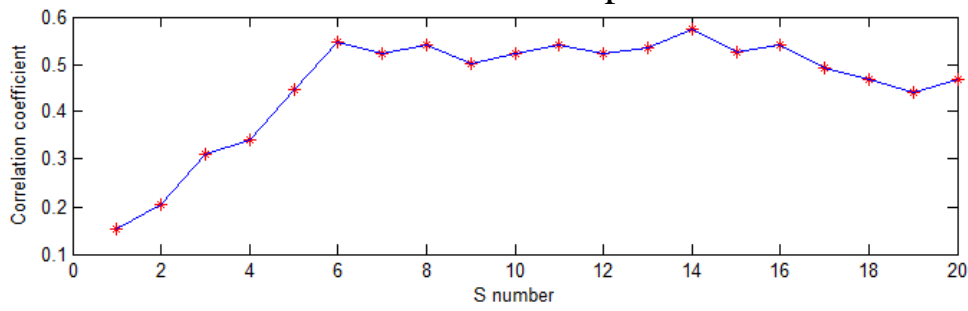

Fig. 2 Influence of S number

As shown in Fig.2,whenS $<6$, the correlation coefficient increasesas $\mathrm{S}$ increases, which there is a large number of extreme value not extracted from the EMD decomposition process. While $6<\mathrm{S}<16$, the correlation coefficient isrelativelystable round 0.5 .After $\mathrm{S}>16$, the correlation coefficient decreases, because the extreme value is affected by the noise in too many loops of EMD.According to the characteristics of PPG signal and the desire of the lowest system power consumption,6is selected to be the value of S.For example,assumethe EEMD loop 10 times and set $S=10$, the algorithm needs to run 40 more times to converge (compare to the case where $\mathrm{S}=6$ ).Therefore, the number of EMD cycles is reduced, which benefitsthe reduction of the system consumption.

In the standard EEMD process, white noise with 0.2 times the intensity of the signal standard deviation is added,which is too much noise to lead the distortion of extracting respiratorywave from PPG.According to the analysisof the PPG signal,the first peak is mainly affected by the frequency component of heart rate,the sub-peak is mainly affected by respiration,blood pressure and other frequency components. When the heart rate is filtered out the amplitude is basicconsistent with the sub-peak of PPG signal as shown in Fig.3.So the average sub-peak amplitude is selected as the noise intensity.

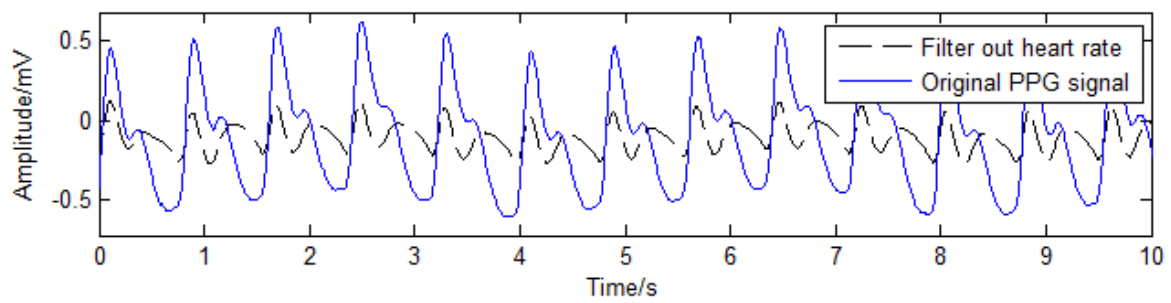

Fig 3Comparison of time domain 


\section{System Architecture}

\section{Front End Circuit}

The digitized PPG can be obtained by the front end circui tof PPG.It contains a PPG capture Circuit, a Low-pass filter and a gain amplifier .

(1)PPG capture circuit

This work adopts a red Light-Emitting Diode whose wavelength is 660nm,an infrared Light-Emitting Diode whose wavelength is $940 \mathrm{~nm}$ and aphotosensitive diode for converting light signals into analog voltage signals.This front-endsensor is used to detect the blood volume changes in finger microvascular. In addition, it can effectively avoid visibleinterference.

(2)A Low-pass filter

Buterworth is selected in this study,because it has the best stable pass-band and stopband. According to the characteristic of PPG signals, the cut-off frequency of the low pass filter is $5 \mathrm{~Hz}$, which can eliminate the visible light,AC power supply noises and improve the running speed of single EMD cycle.

(3)Operational Amplifier

The voltage amplification of the PPG signal is implemented by non-inverting amplifier to be complied with the specification(0-3.3V) of the DSP chip,which is provided by TI Company. In order to protect the DSP chip, the protection circuit is added after the operational amplifier to limit the input amplitude of $\mathrm{A} / \mathrm{D}$ pin below 3.3V.

\section{DSP processor}

The proposed PPG processor comprises a data pre-processing unit, a memory management unit, a sifting process unit and a DSP control unit as shown in Fig. 4.

(1)In order to reduce the complexity of system design, the analog signal is converted into digital signal by using 12 bit Ad converter in DSP. According to the main frequency components of PPG is below $10 \mathrm{~Hz}$,the sampling rate is set to $125 \mathrm{~Hz}$,because high sampling rate consume a large amount of memory and low sampling rate may affect the PPG wave.

(2)The data buffer buffers the input digital signal and sends it to the Sub-peak extraction engine to calculate the average value of the sub-peaks with the extreme value algorithm, simultaneously the extreme points are stored. Then the noise intensity of EEMD is added by multiplying the average value and white noise.

(3)The memory management unit consists of the memory control interface and memory units, which mainly is used to reduce the memory pressure of DSP.

(4)In the sifting process unit the extreme points in the memory is used to the first cycle of EMD.

(5)When the calculation is over, the IMF with the best correlation with the respiration is selected. Based on the best IMF, the respiratory rate is calculated by using the periodic algorithm, and then is displayed on the display unit.

(6)The system control unit selects TMS320F28355 that has high accuracy, low cost, low power consumption and high performance.

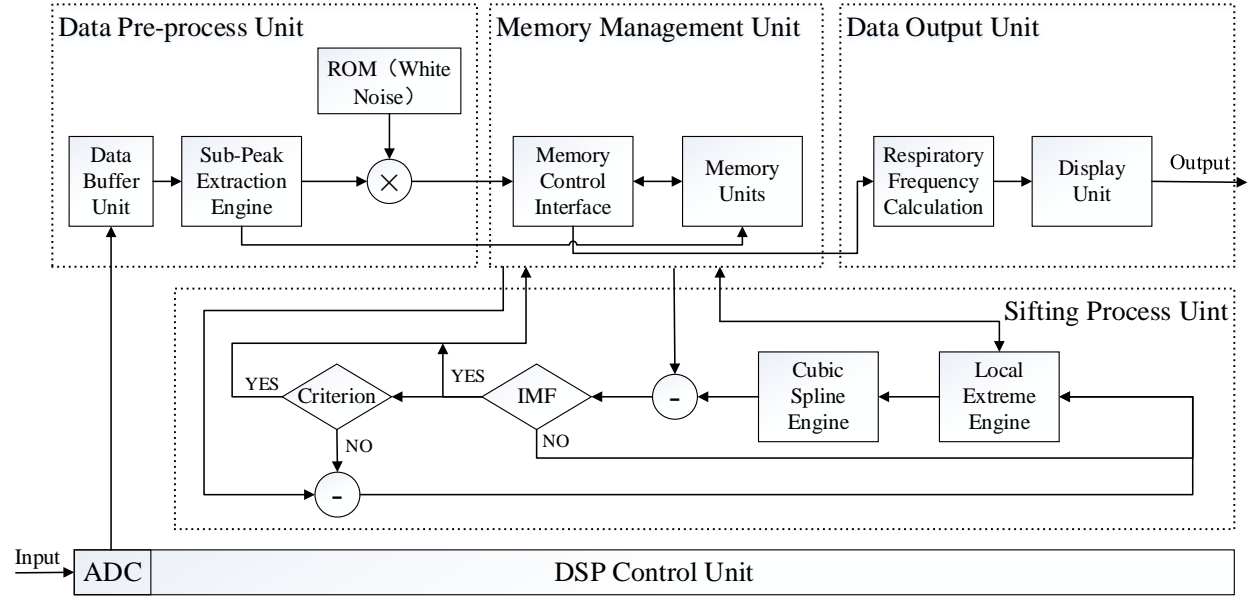

Fig. 4 PPG processor 


\section{SIMULATION AND PHYSICAL RESULTS}

The continuous PPG signals are acquired from a noninvasive near-infrared light sensing device at a sampling rateof $125 \mathrm{~Hz}$.Data points in the first five seconds are taken.The raw data andthe 1 to 7 IMFs resultedfrom theimproved EEMDmethod ispresented in Fig. 5, respectively.It is clear thatIMF7 is highly correlated with respiration and IMF1 is ingeneral correlated with high the frequency noise.

The characteristics of Fourier spectrum ofrespirationand IMF7, as shown in Fig. 6, indicate that the improved EEMD can reconstruct the respiration and inhibit the the noise near the main frequency.

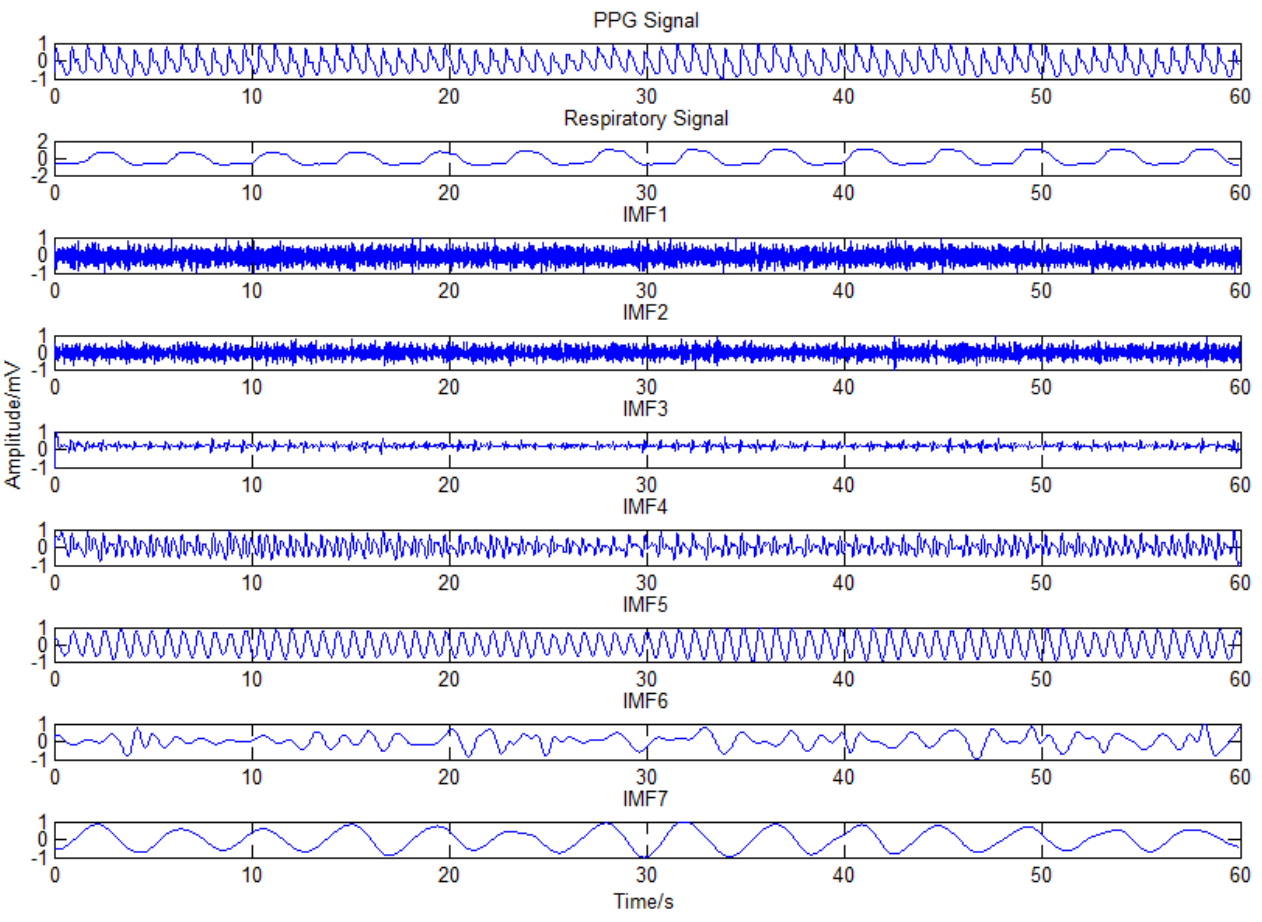

Fig. 5 PPG signals and IMF1-7 by using improved EMD method. Respiratory Signal
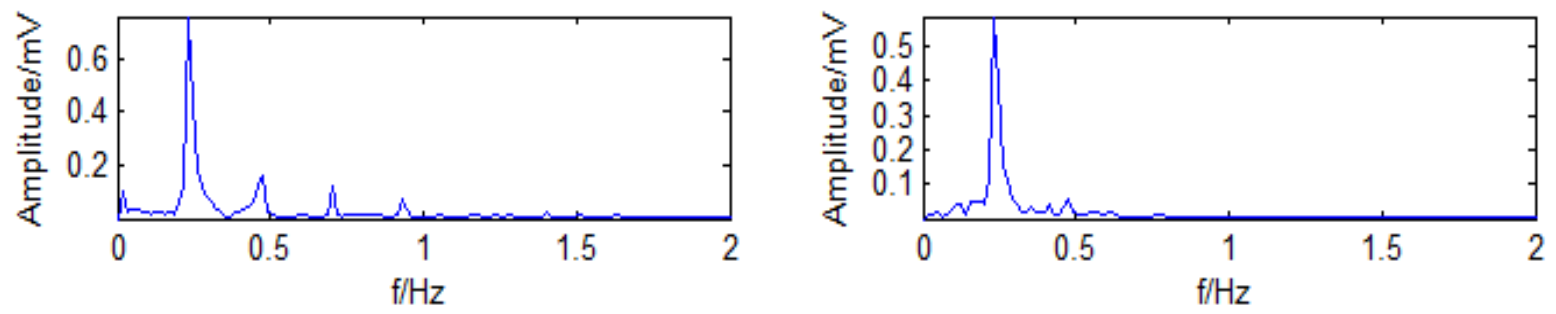

Fig. 6 Fourier Spectrum analysis of respirationand IMF7

In Table 1, L. Wang[5], achieve high sample rate, accurate, low costhardware implementation with five IMFs. EEMD is proposed in [6], which reaches high accuracy performance, but exists problems. The improved EEMD algorithm, our proposed method, is based on the characteristics of the low frequency of the respiration. By using lower sampling rate and higher clock frequency we increased the running speed of the algorithm. The inaccuracy of periodic algorithm increases the error of calculating breathing frequency.

Table 1Comparisonof EMD implementation works

\begin{tabular}{llll}
\hline & {$[5]$} & {$[6]$} & This work \\
\hline Number System & Fixed & Fixed & Floating \\
Signal & ECG & PPG & PPG \\
Algorithm & EMD & EEMD & Improved EEMD \\
IMFs & 5 & 8 & 7 \\
System Freq. & $522.24 \mathrm{KHz}$ & $18.432 \mathrm{MHz}$ & $150 \mathrm{MHz}$ \\
Error & $<5 \times 10^{-3}$ & $\sim 10^{-5}$ & $\sim 10^{-3}$ \\
\hline
\end{tabular}




\section{CONCLUSION}

In this paper, we proposed an effective respiration extraction system, based on the improved EEMD method.The advanced modification makes the hardware implementation applicable more adaptive and low consumption.

\section{References}

[1]Liu xuehong, Wu Aiping. Signal interference in ECG signal based on Wavelet Transform [J], BME \&Clin Med,2003, 78-80(2).

[2]Zouzi run,Chen zhencheng. Extraction of Respiratory Wave from Photoplethysmography Signals[J], Chinese Journal of Biomedical Engineering, 2013, 32(4): 508-512.

[3]N.E.Huang, M.C.Wu.A confidence limit for the empirical mode decomposition and Hilbert spectrum analysis[J], The Royal Society, 2003, 469(10):2317-2345.

[4] Z.H.Wu,N.E.Huang, Ensemble empirical mode decomposition:a noise assisted data analysis method.[J], Advances in Adaptive Data Analysis, 2009, 101 (1): 1-41.

[5]L.Wang,M.IVai,Hardware-acceleratedimplementation of EMD[A], 3rd International Conference on BiomedicalEngineering and Informatics (BMEI)[C],IEEE,2010(2):912-915.

[6]JiaJu Liao,Shang Yi Chuang, An Effective Photoplethysmography Signal Processing System Based on EEMD[A], VLSI Design,Automation and Test (VLSI-DAT)[C], IEEE, 2015:1-4. 